\title{
A New Signal Type Classifier for Fading Environments
}

\author{
Ataollah Ebrahimzadeh and GholamReza Ardeshir \\ Faculty of Electrical and Computer Engineering, Noushirvani Institute of Technology, Babol, Iran
}

\begin{abstract}
Automatic classification of digital signal types is extremely important in communication intelligence. In this paper, we present a highly efficient digital signal type classifier for fading environments. In the proposed method, an equalizer is used to reduce the channel effects. Selected combination of the fourth, the sixth and the eighth order of moments and cumulants of the received signal are introduced as the features for representation of digital signal. A novel multiclass classifier based on support vector machines determines types of the received signals. Simulation results show that the proposed method has a high success rate for recognition of different digital signal types, even at low signal to noise ratio (SNR).
\end{abstract}

Keywords: statistical pattern recognition, signal type classification, fading environments, support vector machine, higher order moments, higher order cumulants

\section{Introduction}

Automatic digital signal type classification (ADSTC) is increasingly important as the number and sophistication of digital signaling systems increase. There is an emerging need for intelligent modems which would be capable of quickly discriminating signal types. Signal type classification may be used to identify interferences or to choose the appropriate demodulator in the cooperative scenario. Due to the increasing usage of digital signal in many novel applications, in this paper we shall focus on classification of these types of signal.

Generally, ADSTC approaches can be divided into two main categories: decision theoretic (DT) approaches and pattern recognition (PR) approaches. DT approaches use probabilistic and hypothesis testing arguments to formulate the recognition problem [1-7]. The major drawbacks of DT approaches are computational complexity, difficulty of their implementation and thorough analysis required to set the proper threshold values [8]. However, PR techniques do nut need such a careful treatment. PR techniques are divided into two main subsystems: the feature extraction subsystem and the classifier subsystem [8-20]. The former is responsible for extracting prominent characteristics of the received signal, and the latter determines the class of the signal.

Most of the automatic digital signal type classifiers have been proposed for recognition of signal formats in additive white Gaussian noise (AWGN) channels [1-5], [8-17]. However, in real world, communication environments, such as wireless communication channels suffer from fading. There are few ADSTC methods over fading environments $[6,7],[18-20]$. In [6], a method is proposed for classification of PSK2 and PSK4 in a flat Rayleigh fading. In [7], a quasi-optimal solution based on the approximation of the log-likelihood function is proposed to identify FSK signals in a fading channel. In [18], signal formats PSK2, QPSK4, $\pi / 4$ QPSK and QAM16 were classified by applying the nearest neighbor rule in a two-dimensional feature space, i.e. the variance of differential phase between consecutive symbols and standard deviation of the instantaneous amplitude. In [19], a method has been proposed based on a combination of the fourth order cumulants and power moment matrices for identification of PSK2, PSK4, QAM8, ASK2 and ASK4 digital signals in multipath environment. In this method a multi-layer perceptron (MLP) neural network is used as the classifier. In [20], a SVMs-based classifier is used for identification of PSK2, PSK4, PSK8, ASK2 and ASK4. In this method a combination of the fourth order cumulants and symmetry of the received signal are used as the features. 
In this paper, we present a highly efficient digital signal type classifier (HEDSTC) for fading environments. Figure 1 shows the general scheme of HEDSTC. It includes four main modules. In the preprocessing module, components of the channel noise that are outside the signal bandwidth are removed, carrier frequency is estimated, complex envelope is recovered, etc. This module is similar to ADSTC methods and will not be explained here. The role of equalization module is to reduce the channel distortion. Feature extraction module extracts a selected combination of the higher order moments and higher order cumulants up to eighth. Classifier module has a multiclass structure based on binary support vector machines (SVMs).

The paper is organized as follows. In Section 2 , the channel equalization will be explained. The feature extraction module will be described in Section 3. The multi-class support vectors machine classifier (as the new proposed classifier) is explained in Section 4. Section 5 shows some of the simulation results. Finally, Section 6 concludes the paper.

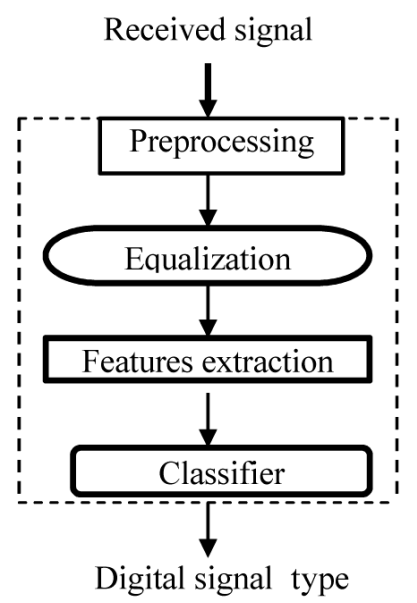

Figure 1. General scheme of HEDSTC.

\section{Channel Equalization}

In digital communications, there are four digital modulation techniques based on changes of frequency, amplitude, phase of message, frequency shift keying (FSK), amplitude shift keying (ASK), phase shift keying (PSK) and quadrature amplitude modulation (QAM) [21]. In real world scenarios, the transmission channel is a critical factor that may cause unrecoverable distortions on the signal, particularly for higher order digital signals. To reduce the dispersion effects of channel, an equalization stage has been used in the receiver. In applications such as signal type classification, the training set which is needed for adjusting the coefficients of equalizer is not available. Hence the equalization must be done blindly. When the type of signal is unknown, the fractionally spaced equalizer-constant modulus algorithm (FSE-CMA) is one of the common blind equalization algorithms [22]. FSE-CMA is the integration of two different parts: the constant modulus algorithms (CMA) and the fractional spaced equalizer (FSE).

The constant modulus algorithm (CMA) is a stochastic gradient algorithm, designed to force the equalizer weight to keep a constant envelope. Even though it was originally designed for problems where the signal of interest has a constant envelope property, it has been shown that it works well for some of QAM types [22]. As a result, the CMA is expected to have better performance for FSK and PSK signals than QAM types. The CMA cost function is given by:

$$
J(k)=E\left\{|y(k)|^{2}-\gamma\right\}
$$

where $y(k)$ is the equalizer output and $\gamma$ called the dispersion constant that defined by (2).

$$
\gamma=\frac{M_{40}}{M_{20}}
$$

where $M_{40}$ and $M_{20}$ are fourth and second order moment respectively. The cost function $J(k)$ is minimized iteratively by using a gradient-based algorithm.

In any standard CMA equalization system, the coefficient taps are baud-spaced. However, it is often desired to use an equalizer with taps spaced at a fraction of the data symbol period $\mathrm{T}$. This configuration gives extra degrees of freedom to perform additional filtering. Such a scheme is called fractional spaced equalization (FSE). Let us assume that the received signal is:

$$
x(t)=\sum_{k=-\infty}^{\infty} s(k) h(t-k T)+v(t)
$$

where $h(t)$ is the channel impulse response, $s(k)$ is the sequence of information and $v$ is AWGN. 
It is assumed that $h(t)$ has finite length. Fractionally spaced channel output which results from $P$ times oversampling with respect to symbol rate can be written as:

$$
x\left(k \frac{T}{P}\right)=\sum_{l=-\infty}^{\infty} s(l) h\left(k \frac{T}{P}-l T\right)+v\left(k \frac{T t}{P}\right) .
$$

An equivalent representation can be formed by using P-channel parallel filter bank model. The output of the $i^{t h}$ sub-channel $h_{i}(k)$ is given by:

$$
\begin{gathered}
x_{i}(k)=\sum_{l=-\infty}^{\infty} s(l) h_{i}(k-l)+v_{i}(k) ; \\
i=0, \ldots, P-1 .
\end{gathered}
$$

Now, we use an equalizer $w_{i}(k)$ in cascade with each sub-channel $h_{i}(k)$. The equalizer coefficients (taps) are adjusted by using FSE-CMA algorithm:

$$
w(k+1)=w(k)+\zeta x^{*}(k) y(k)\left(|y(k)|^{2}-\gamma\right)^{2}
$$

where $\zeta$ is the step size parameter and:

$$
\begin{aligned}
x(k)= & {\left[x^{1}(k), \ldots, x^{1}(k-(N-1)), \ldots,\right.} \\
& x^{P}(k), \ldots, x^{P}(k-(k-(N-1))]^{T} .
\end{aligned}
$$

\section{Feature Extraction}

Different digital signal types have different characteristics. Therefore, extracting proper features for recognition of digital signals, particularly in the case of higher order and/or nonsquare types, is a serious problem. Choosing unsuitable features can make it impossible, even for an advanced classifier, to perform a simple task, while choosing appropriate features can make it possible for a simple classifier to solve complex problems [23].

In this paper, we consider the following digital signal types: PSK2, PSK4, PSK8, ASK8, QAM32, V29, Star-QAM8 and QAM64. To simplify the indication, we use $\mathrm{P}_{1}, \mathrm{P}_{2}, \mathrm{P}_{3}, \mathrm{P}_{4}$, $\mathrm{P}_{5}, \mathrm{P}_{6}, \mathrm{P}_{7}$ and $\mathrm{P}_{8}$ to denote PSK2, PSK4, PSK8, ASK8, QAM32, V29, Star-QAM8 and QAM64 respectively. Among different features that have been extracted, the higher order moments and higher order cumulants up to eighth, achieve the highest performances in classification of the considered digital signals. The following subsections briefly describe these features.

\subsection{Moments}

Probability distribution moments are the generalization of the concept of the expected value. Recall that the general expression for the $i^{t h}$ moment of a random variable is given by [24]:

$$
\mu_{i}=\int_{-\infty}^{\infty}(s-m)^{i} f(s) d s
$$

where $m$ is the mean of the random variable. The definition of the $i^{\text {th }}$ moment for a finite length discrete signal is given by:

$$
\mu_{i}=\sum_{k=1}^{N}\left(s_{k}-m\right)^{i} f\left(s_{k}\right)
$$

where $N$ is the data length. In this study, signals are considered to be zero mean. Thus:

$$
\mu_{i}=\sum_{k=1}^{N} s_{k}^{i} f\left(s_{k}\right)
$$

Now, the auto-moment of the random variable may be defined as follows:

$$
M_{p q}=E\left[s^{p-q}\left(s^{*}\right)^{q}\right]
$$

where $p$ is the moment order and $s^{*}$ stands for complex conjugation $s$.

Consider a zero mean discrete signal sequence with the form $s=a+j b$. Using the definition of the auto-moments, the expressions for different orders may be easily derived. For example:

$$
M_{41}=E\left[(a+j b)^{3}(a-j b)\right]=E\left[a^{4}-b^{4}\right] .
$$

\subsection{Cumulants}

Consider a scalar zero mean random variable $s$ with characteristic function:

$$
\hat{f}(t)=E\left\{e^{j t s}\right\} .
$$

Expanding the logarithm of the characteristic function as a Taylor series, we have:

$$
\log \hat{f}(t)=k_{1}(j t)+\frac{k_{2}(j t)^{2}}{2}+\ldots+\frac{k_{r}(j t)^{r}}{r !}+\ldots
$$


The constants $k_{i}$ in (14) are called the cumulants. The symbolism for $p^{\text {th }}$ order of cumulants is similar to the $p^{\text {th }}$ order moment. More specifically:

$$
C_{p q}=\operatorname{Cum}[\underbrace{s, \ldots, s}_{(p-q) \text { terms }}, \underbrace{s^{*}, \ldots, s^{*}}_{(q) \text { terms }}] .
$$

For example:

$$
C_{81}=\operatorname{Cum}\left(s, s, s, s, s, s, s, s^{*}\right) .
$$

\subsection{Relations between Moments and Cumulants}

The $n^{\text {th }}$ order cumulant is a function of the moments of orders up to $n$. Moments can be expressed in terms of cumulants as:

$$
\begin{aligned}
& M\left[s_{1}, \ldots, s_{n}\right]= \\
& \sum_{\forall v} \operatorname{Cum}\left\lfloor\left\{s_{j}\right\}_{j \in v_{1}}\right\rfloor \ldots u m\left\lfloor\left\{s_{j}\right\}_{j \in v_{1}}\right\rfloor
\end{aligned}
$$

where the summation index is over all partitions $v=\left(v_{1}, \ldots, v_{q}\right)$ for the set of indices $(1,2, \ldots, n)$, and $q$ is the number of elements in a given partition. Cumulants can be also derived in terms of moments. The $n^{\text {th }}$ order cumulant of a discrete signal $s(n)$ is given by:

$$
\begin{aligned}
& \operatorname{Cum}\left[s_{1}, \ldots, s_{n}\right]= \\
& \sum_{\forall v}(-1)^{q-1}(q-1) ! E\left[\prod_{j \in v_{1}} s_{j}\right] \ldots E\left[\prod_{j \in v_{q}} s_{j}\right]
\end{aligned}
$$

where the summation is performed on all partitions $v=\left(v_{1}, \ldots, v_{q}\right)$ for the set of indices $(1,2, \ldots, n)$.

Let $n=3$. In this case, the available set of indices is $(1,2,3)$, and four different types of partitioning may be obtained for that set: $\{(1,2,3)\}$ leads to $q=1,\{(1),(2,3)\}$ leads to $q=2,\{2,(1,3)\}$ leads to $q=2,\{3,(1,2)\}$ leads to $q=2,\{(1),(2),(3)\}$ leads to $q=3$.
Therefore:

$$
\begin{aligned}
\operatorname{Cum}\left[s_{1}, s_{2}, s_{3}\right] & =(-1)^{1-1}(1-1) ! E\left[s_{1} s_{2} s_{3}\right] \\
+ & (-1)^{2-1}(2-1) ! E\left[s_{1}\right] E\left[s_{2} s_{3}\right] \\
+ & (-1)^{2-1}(2-1) ! E\left[s_{2}\right] E\left[s_{1} s_{3}\right] \\
+ & (-1)^{2-1}(2-1) ! E\left[s_{3}\right] E\left[s_{1} s_{2}\right] \\
& +(-1)^{2-1}(2-1) ! E\left[s_{3}\right] E\left[s_{1} s_{2}\right] \\
& +(-1)^{3-1}(3-1) ! E\left[s_{1}\right] E\left[s_{2} s_{3}\right],
\end{aligned}
$$

$$
\begin{aligned}
\operatorname{Cum}\left[s_{1}, s_{2}, s_{3}\right] & =E\left[s_{1} s_{2} s_{3}\right]-E\left[s_{1}\right] E\left[s_{2} s_{3}\right] \\
& +E\left[s_{2}\right] E\left[s_{1} s_{3}\right]-E\left[s_{3}\right] E\left[s_{1} s_{2}\right] \\
& +2 E\left[s_{1}\right] E\left[s_{2}\right] E\left[s_{3}\right] .
\end{aligned}
$$

Using the same manner, cumulants expressions up to eighth order can be computed. Among these features, we need to select the best ones. The best selected features are: $\mathrm{C}_{40}, \mathrm{M}_{41}, \mathrm{M}_{61}$, $\mathrm{C}_{61}, \mathrm{C}_{63}, \mathrm{M}_{84}, \mathrm{C}_{80}, \mathrm{C}_{82}$ and $\mathrm{C}_{83}$. Table 1 shows some of these features for a number of considered digital signal types. These values are computed under the constraint of unit variance and are noise free.

\begin{tabular}{|c||c|c|c|c|c|}
\cline { 2 - 6 } \multicolumn{1}{c||}{} & $\mathrm{P}_{1}$ & $\mathrm{P}_{2}$ & $\mathrm{P}_{4}$ & $\mathrm{P}_{6}$ & $\mathrm{P}_{7}$ \\
\hline \hline$M_{41}$ & 1 & 0 & 1.76 & 0 & 0 \\
\hline$M_{61}$ & 1 & -1 & 3.62 & 8.667 & 2.92 \\
\hline$C_{63}$ & 16 & 4 & 7.19 & -4.43 & 0.160 \\
\hline$M_{84}$ & 1 & 1 & 7.92 & 28.75 & 5.25 \\
\hline$C_{83}$ & -244 & 0 & 9.27 & 0 & 0 \\
\hline
\end{tabular}

Table 1. Some of the chosen features for a number of considered digital signal types.

\section{Classifier}

We have proposed a multi-class SVM-based classifier. The SVMs are based on structural risk minimization (SRM) principle that lets them have high generalization ability and several applications in the area of pattern recognition [25]. Though SVM was originally designed for two-class problems, several approaches have been proposed to extend SVM for multi-class data sets [26]. The following subsections describe briefly the binary SVM and multi-class classifier. 


\subsection{Binary SVM (BSVM)}

BSVM constructs optimal separating hyperplanes (OSH) for classification. OSH maximizes the margin between the two nearest data points belonging to two classes. The idea of BSVM can be expressed as follows.

Consider the training set $\left(x_{i}, y_{i}\right), i=1,2, \ldots, l$, $x \in R^{d}, y \in\{-1,+1\}$ which can be separated by the hyperplane $w^{T} x+b=0$, where $w$ is the weight vector and $b$ is the bias. If this hyperplane maximizes the margin, then the following inequality is valid for all input data:

$$
y_{i}\left(\mathbf{w}^{T} \mathbf{x}_{i}+b\right) \geq 1 \text { for all } \mathbf{x}_{i}, i=1,2, \ldots, l .
$$

The margin of the hyperplane is equal to $2 /\|w\|$. Thus, the problem is to maximize the margin by minimizing $\|w\|^{2}$ subject to (20). This is a convex quadratic programming $(\mathrm{QP})$ problem. To solve this problem, Lagrange multipliers $\left(\alpha_{1}, i=1, \ldots, l ; \alpha_{1} \geq 0\right)$ are used:

$$
L_{p}=\frac{1}{2}\|\mathbf{w}\|^{2}-\sum_{i=1}^{l} \alpha_{i}\left[y_{i}\left(\mathbf{w}^{T} \mathbf{x}_{i}+b\right)-1\right] .
$$

After minimizing $L_{P}$ in terms of both $w$ and $b$, the optimal weights are given by:

$$
\mathbf{w}^{*}=\sum_{i=1}^{l} \alpha_{i}^{*} y_{i} \mathbf{x}_{i}
$$

The dual of the problem is given by:

$$
L_{d}=\sum_{i=1}^{l} \alpha_{i}-\frac{1}{2} \sum_{i=1}^{l} \sum_{j=1}^{l} \alpha_{i} \alpha_{j} y_{i} y_{j} \mathbf{x}_{i}^{T} \mathbf{x}_{j}
$$

$L_{d}$ should be maximized under the constraints of $\sum_{i=1}^{l} \alpha_{i} y_{i}=0$. The Lagrange multipliers are only non-zero when $y_{i}\left(\mathbf{w}^{T} \mathbf{x}_{i}+b\right)=1$. The training points, for which the equality of (20) holds, are called support vectors (SV) that can satisfy $\alpha_{i} \prec 0$. The optimal bias for any support vector $\mathbf{x}_{i}$ is given by:

$$
b^{*}=y_{i}-\mathbf{w}^{* T} \mathbf{x}_{i} .
$$

Optimal decision function (ODF) is given by:

$$
f(\mathbf{x})=\operatorname{sgn}\left(\sum_{i=1}^{l} y_{i} \alpha_{i}^{*} \mathbf{x}^{T} \mathbf{x}_{i}+b^{*}\right)
$$

where $\alpha_{i}^{*}$ 's are optimal Lagrange multipliers.
For the input data with high noise levels, SVM uses soft margins that can be expressed as follows, with the introduction of the non-negative slack variables $\xi_{i}, i=1, \ldots, l$ :

$$
y_{i}\left(w^{T} x_{i}+b\right) \geq 1-\xi_{i} \text { for } i=1,2, \ldots, l .
$$

To obtain the OSH, the $\Phi=\frac{1}{2}\|\mathbf{w}\|^{2}+C \sum_{i=1}^{l} \xi_{i}{ }^{k}$ should minimize the subject to (26), where $C$ is the penalty parameter. The parameter $C$ controls tradeoff between the complexity of the decision function and the number of training examples which are misclassified.

In the nonlinearly separable cases, SVM maps the training points to a high dimensional feature space using the kernel function $K\left(\vec{x}_{i}, \vec{x}_{j}\right)$, where linear separation can be possible. One of the most famous kernel functions is Gaussian radial basis function (GRBF) given by:

$$
K(x, y)=\exp \left(-\|x-y\|^{2} / 2 \sigma^{2}\right)
$$

where $\sigma$ is the width of the RBF kernel. After selecting the kernel function, the QP problem is:

$$
L_{d}=\sum_{i=1}^{l} \alpha_{i}-\frac{1}{2} \sum_{i=1}^{l} \sum_{j=1}^{l} \alpha_{i} \alpha_{j} y_{i} y_{j} K\left(\mathbf{x}_{i}, \mathbf{x}_{j}\right)
$$

where $\alpha_{i}^{*}$ is derived by:

$$
\begin{aligned}
& \alpha_{i}^{*}=\arg \max _{\alpha} L_{d} \\
& 0 \leq \alpha_{i} \leq C ; i=1,2, \ldots, l ; \sum_{j=1}^{l} \alpha_{i} y_{i}=0 .
\end{aligned}
$$

After training, we have the following decision function:

$$
f(\mathbf{x})=\operatorname{sgn}\left(\sum_{i=1}^{l} y_{i} \alpha_{i}^{*} K\left(\mathbf{x}, \mathbf{x}_{i}\right)+b^{*}\right) .
$$

\subsection{Multi-class SVM-based Classifier}

In this paper, we propose a simple method for combining the binary SVMs, to construct a multi-class classifier. We use an approach similar to the one in [27]. Our approach can be described as follows: 
Let $\left\{P_{i}: i=1,2, \ldots, N\right\}$ be $N$ classes of signals. We construct $N$ classifiers $\left\{f_{i}: i=\right.$ $1,2, \ldots, N\}$ and each classifier is trained by the method of one-class-versus-the-rest; that is, the classifier $f_{i}$ is trained for $P_{i}$ versus the rest of the classes. Then, in the signal classification phase, the classifiers make decision according to the following decision rule:

$$
x \Rightarrow P_{i} \text { if } f_{i}=\max \left\{f_{k}(x)>0 ; k=1, \ldots, N-1\right\}
$$

where the function $f_{k}(x)$ provides the distance of $x$ to the decision surfaces.

\section{Simulation Results}

In this section, we evaluate the performance of HEDSTC for identification of the considered digital signal types. We assumed that carrier frequencies were estimated correctly and the signals heterodyned down. Thus, the only complex base-band signals were considered. For each digital signal type modulation scheme 10000 samples were created and stored. Simulations studies were done by using the channel models developed in [28].

First, a 6000-sample sequence was extracted out of the 10000 samples generated for each digital signal type. Next, each sequence was passed through propagation channel. Then the resulting signal sequences were corrupted with additive white Gaussian noise with SNR levels between 0 to $20 \mathrm{~dB}$. For FSE-CMA algorithm, a 12-tap equalizer was chosen and the step size was set to 0.5 to ensure that the algorithm is stable.

Finally, 200 trials per SNR level were generated. We used multiple trials per SNR level to get a sense of the variance in the measurements and enhance the performances of the classifier. Next, the features were extracted from each noisy signal. These features fed into the SVMs for training. After training, we tested the trained classifier. For testing phase we selected seven SNR levels, i.e. $2 \mathrm{~dB}, 5 \mathrm{~dB}, 8 \mathrm{~dB}, 11 \mathrm{~dB}, 14 \mathrm{~dB}$, $17 \mathrm{~dB}$ and $20 \mathrm{~dB}$. In each test, a new random message and noise was created to ensure the independence of all results. Classifying by the new SVM-based classifier, GRBF kernel function showed better performance than the others. Hence we used GRBF in all of SVMs. The kernel parameters for all of SVMs were chosen $\sigma=1$, based on trial and error method. We evaluated the performance of HEDSTC in two stages described in the following subsections.

\subsection{Performance of HEDSTC in Typical Urban Environment}

In this subsection, we consider a typical urban environment as the propagation environment. The mobile speed is considered $85 \mathrm{~km} / \mathrm{h}$. Tables 2-4 show the confusion matrices of the HEDSTC at three levels of SNR, i.e. $2 \mathrm{~dB}, 8 \mathrm{~dB}$ and $17 \mathrm{~dB}$.

\begin{tabular}{|c||c|c|c|c|c|c|c|c|} 
& $\mathrm{P}_{1}$ & $\mathrm{P}_{2}$ & $\mathrm{P}_{3}$ & $\mathrm{P}_{4}$ & $\mathrm{P}_{5}$ & $\mathrm{P}_{6}$ & $\mathrm{P}_{7}$ & $\mathrm{P}_{8}$ \\
\hline \hline $\mathrm{P}_{1}$ & 91 & & 8 & 1 & & & & \\
\hline $\mathrm{P}_{2}$ & 8 & 84 & 4 & & & 1 & & 3 \\
\hline $\mathrm{P}_{3}$ & 14 & 8 & 78 & & & & & \\
\hline $\mathrm{P}_{4}$ & 11 & 3 & & 86 & & & & \\
\hline $\mathrm{P}_{5}$ & & & & & 76 & & 4 & 20 \\
\hline $\mathrm{P}_{6}$ & 2 & 1 & & & & 78 & & 19 \\
\hline $\mathrm{P}_{7}$ & & 5 & & & 20 & & 75 & \\
\hline $\mathrm{P}_{8}$ & & & & & 4 & 25 & & 71 \\
\hline
\end{tabular}

Table 2. Confusion matrix of HEDSTC in $\mathrm{SNR}=2 \mathrm{~dB}$.

\begin{tabular}{|c||c|c|c|c|c|c|c|c|}
\cline { 2 - 9 } \multicolumn{1}{l||}{} & $\mathrm{P}_{1}$ & $\mathrm{P}_{2}$ & $\mathrm{P}_{3}$ & $\mathrm{P}_{4}$ & $\mathrm{P}_{5}$ & $\mathrm{P}_{6}$ & $\mathrm{P}_{7}$ & $\mathrm{P}_{8}$ \\
\hline \hline $\mathrm{P}_{1}$ & 100 & & & & & & & \\
\hline $\mathrm{P}_{2}$ & 4 & 96 & & & & & & \\
\hline $\mathrm{P}_{3}$ & 6 & 1 & 93 & & & & & \\
\hline $\mathrm{P}_{4}$ & 2 & & & 98 & & & & \\
\hline $\mathrm{P}_{5}$ & & & & & 90 & & 1 & 9 \\
\hline $\mathrm{P}_{6}$ & & & & & & 91 & & 9 \\
\hline $\mathrm{P}_{7}$ & & & & & 12 & & 88 & \\
\hline $\mathrm{P}_{8}$ & & & & & 1 & 12 & & 87 \\
\hline
\end{tabular}

Table 3. Confusion matrix of HEDSTC in $\mathrm{SNR}=8 \mathrm{~dB}$.

\begin{tabular}{|l||c|c|c|c|c|c|c|c|}
\multicolumn{1}{l||}{} & $\mathrm{P}_{1}$ & $\mathrm{P}_{2}$ & $\mathrm{P}_{3}$ & $\mathrm{P}_{4}$ & $\mathrm{P}_{5}$ & $\mathrm{P}_{6}$ & $\mathrm{P}_{7}$ & $\mathrm{P}_{8}$ \\
\hline \hline $\mathrm{P}_{1}$ & 100 & & & & & & & \\
\hline $\mathrm{P}_{2}$ & & 100 & & & & & & \\
\hline $\mathrm{P}_{3}$ & & & 100 & & & & & \\
\hline $\mathrm{P}_{4}$ & & & & 100 & & & & \\
\hline $\mathrm{P}_{5}$ & & & & & 97 & & & 3 \\
\hline $\mathrm{P}_{6}$ & & & & & & 98 & & 2 \\
\hline $\mathrm{P}_{7}$ & & & & & 4 & & 96 & \\
\hline $\mathrm{P}_{8}$ & & & & & & 6 & & 94 \\
\hline
\end{tabular}

Table 4. Confusion matrix of HEDSTC in SNR $=17 \mathrm{~dB}$. 
Figure 2 shows the probability of the correct classification $\left(\mathrm{P}_{C}\right)$ of HEDSTC at different SNRs. Note that $\mathrm{P}_{c}$ at each SNR is equal to the average value of the numbers that appear in the diagonal of the confusion matrix. It can be seen that HEDSTC classifies the digital signal types with high accuracy. Basically, this is due to the selected features and the proposed classifier. The selected features have effective properties in signal representation. On the other hand, the proposed classifier has high generalization ability.

In Section 2, we explained FSE-CMA. In order to evaluate the contribution of the equalizer, the classification results for non-equalized and equalized signals are compared. Figure 3 and Figure 4 show these results for PSK8 and QAM64 signals, respectively. It can be seen that performances of the classifier with equalizer are higher than performances of the classifier with-

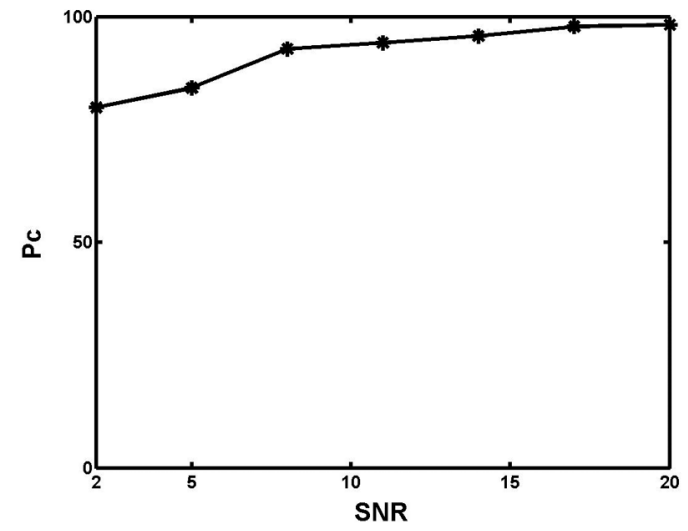

Figure 2. Performance of HEDSTC at different SNRs.

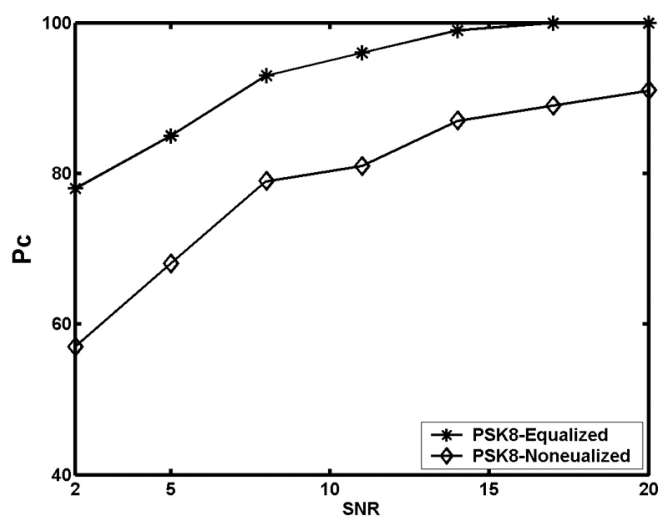

Figure 3. Classification results for equalized and non-equalized PSK8.

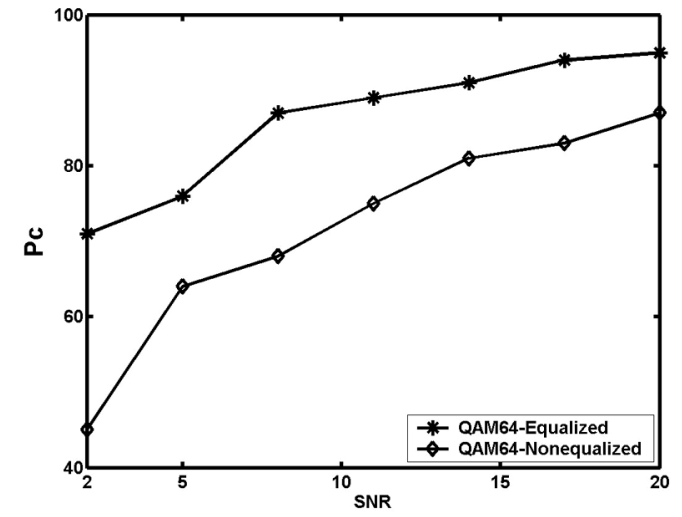

Figure 4. Classification results for equalized and non-equalized QAM 64.

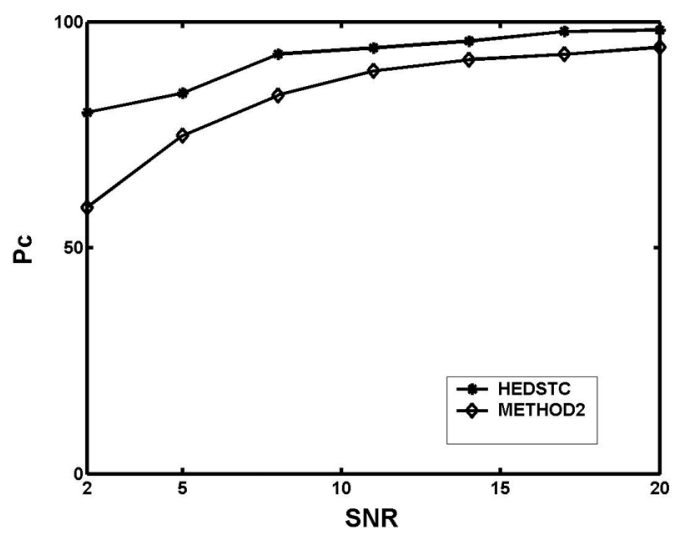

Figure 5. Comparison between the performances of HEDSTC and METHOD2 at different SNRs.

out equalizer, both for PSK8 and QAM64 SIGNALS. Therefore it can be said that equalizer plays an important role in the classification of digital signals over fading environments.

In order to indicate the effectiveness of the proposed classifier, we consider a radial basis function neural network (RBFNN), which has high performance in neural networks [29]. The set up simulations are the same. We name this method METHOD2. Figure 5 shows performances of two classifiers in different SNRs. It can be seen that HEDSTC has higher accuracy than METHOD2 at all SNRs, particularly at low SNRs. When the SNR is low, METHOD2 shows poor performance while in higher SNRs its success rate is higher. The reason may be that in low SNRs the structure of neural network is not proper and sometimes it will not converge, which in turn results in low generali- 
zation ability. In higher SNRs, the features are proper and closer to the noiseless state and it is easier to construct the neural network results in high classification probability. The SVM classifier sufficiently uses the information of features and maps them nonlinearly into a high dimensional feature space and constructs the optimum separating hyperplane to make the data linearly separable. Thus, the probability of correct classification is increased and HEDSTC provides excellent performance of classification of considered digital signals at all of SNRs.

In order to show the effectiveness of the extracted features, we have used the features introduced in [8]. The structure of the classifiers and the simulations setup are the same. We name this method METHOD3. Figure 6 shows the performances of METHOD3 and HEDSTC at different SNRs. Results imply that our selected features have effective properties in signal representation.

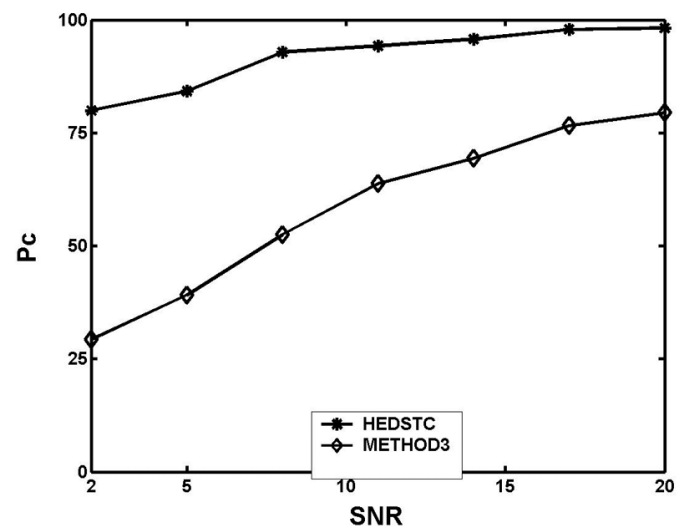

Figure 6. Performances of HEDSTC and METHOD3 at different SNRs.

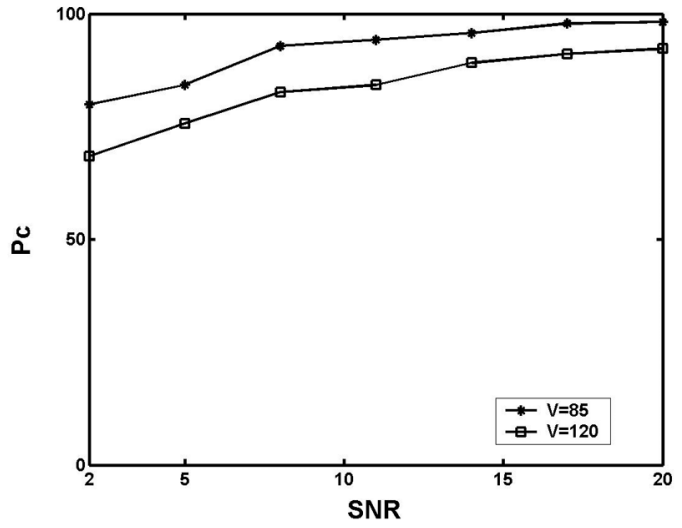

Figure 7. Performances of HEDSTC at different speeds.
In order to evaluate the performance of HEDSTC at different mobile speeds, we have changed the mobile speed to $120 \mathrm{~km} / \mathrm{h}$. Figure 7 shows the classification results of HEDSTC at $20 \mathrm{~km} / \mathrm{h}$ as well as at $85 \mathrm{~km} / \mathrm{h}$. It can be seen at 120 $\mathrm{km} / \mathrm{h}$, the performance of HEDSTC is lower than the performance of HEDSTC in case of 85 $\mathrm{km} / \mathrm{h}$; however it does not degrade drastically.

\subsection{Performance of HEDSTC in Different Propagation Environments}

In this subsection we experiment with the performances of HEDSTC in different propagation environments, i.e. rural and bad urban environments. The mobile speed is considered to be 85 $\mathrm{km} / \mathrm{h}$. Figure 8 shows the classification results of HEDSTC in these environments. Also, the performance of HEDSTC in the typical urban is showed. It is found that HEDSTC performs classification task very well, even in bad urban environment. Although the performance of HEDSTC in rural environment is better than in other environments, it can be said that HEDSTC has nearly robust performance in different fading environments.

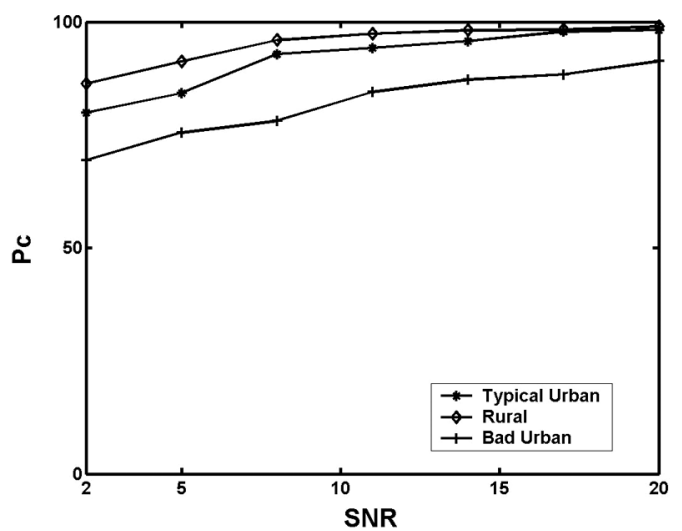

Figure 8. Performances of HEDSTC in different fading environments.

\section{Conclusion}

Automatic digital signal type classification is the most important topic in communication intelligence. Most of the previous methods have been proposed for classification of digital signals in AWGN environments. However, in real 
world, we face the fading environments. This paper presents a highly efficient digital signal type classifier for fading environments. In this method the channel dispersion is mitigated using an FSE-CMA equalizer. A simple multiclass SVM-based classifier is proposed to determine the membership of the received signal. A selected combination of the higher order moments and higher order cumulants are proposed for representation of the digital signals. Simulation results show that the proposed method works very well, even at low SNRs and shows a nearly robust performance in different conditions. For future works, we can change model of the fading environments and experiment with the proposed method in these environments. Also, we can consider another classifier and compare the respective results with the results presented in this paper.

\section{Acknowledgment}

The authors would like to thank R\&D office of TCKH, especially Mr. Eng. Sahbaee, for funding and encouraging this work.

\section{References}

[1] C. Long, K. Chugg, A. Polydoros, Further results in maximum likelihood classification of QAM signals. Proc. MILCOM, (1994), pp. 57-61.

[2] C. L. Martret, D. BoiteA, A general maximum likelihood classifier for modulation classification. Proc. ICASSP, (1998), pp. 2165-2168.

[3] P. Panagotiou, A. Polydoros, Likelihood ratio tests for modulation classifications. Proc. MILCOM, (2000), pp. 670-674.

[4] W. WEI, J. M. MENDEL, Maximum-likelihood classification for digital amplitude-phase modulations. IEEE Trans. Comm., 48 (2000), pp. 189-193.

[5] K. M. ChugG, C. S. Long, A. Polydoros, Combined likelihood power estimation and multiple hypothesis modulation classification. Proc. 29 Asilomar Conf., (1995), pp. 1137-1141.

[6] L. HonG, K. C. Ho, BPSK and QPSK modulation classification with unknown signal level. Proc. MILCOM, (2000), pp. 976-980.

[7] A. E. El-Mahdy, N. M. NAmAzi, Classification of multiple M-ary frequency-shift keying signals over a Rayleigh fading channel. IEEE Trans. Comm, 50 (2002), pp. 967-974.
[8] A. Swami, B. M. SADLER, Hierarchical digital modulation classification using cumulants. IEEE Trans. Comm., 48 (2000), pp. 416-429.

[9] A. K. NANDI, E. E. Azzouz, Algorithms for automatic modulation recognition of communication signals. IEEE Trans. Comm., 46 (1998), pp. 431436.

[10] B. G. MobASSERI, Digital modulation classification using constellation shape. Signal Processing, 80 (2000), pp. 251-277.

[11] A. O. Hero, H. H. Mahram, Digital modulation classification using power moment matrices. Proc. ICASSP, (1998), pp. 3285-3288.

[12] M. Pedzisz, A. Mansour, Automatic modulation recognition of digital signals using constellation rotation. Digital Signal Processing, 15 (2005), pp. 295-304.

[13] S. Z. Hsue, S. S. Soliman, Automatic modulation classification using Zero-Crossing. IEE Proc. Radar, Sonar and Navigation, 137 (1990), pp. 459-464.

[14] K. C. Ho, W. Prokopiw, Y. T. Chan, Modulation identification of digital signals by wavelet transform. IEE Proc. Radar, Sonar and Navigation, 147 (2000), pp. 169-176.

[15] C. L. P. SEHIER, Automatic modulation recognition with a hierarchical neural network. Proc. MILCOM, (1993), pp. 111-115.

[16] L. Mingquan, X. Xianci, L. Leming, Cyclic spectral features-based modulation recognition. Proc. Comm. Tech., 2 (1998), pp. 792-795.

[17] A. EBRAhimzadeH, S. A. SEYYedin, A new method for automatic digital signal type identification. Proc. CIS, (2005).

[18] H. YoshIOKA, A fast modulation recognition technique using nearest neighbor rules with optimized threshold for modulation classification in Rayleigh fading channels. Proc. WPMC, (2002), pp. 10491052.

[19] A. EBRAhimzadeH, S. A. SEYYedin, A new method for modulation identification in fading environments. Proc. CIS, (2005).

[20] A. Ebrahimzadeh, S. A. Seyyedin, Automatic digital signal classification using SVM in fading environments. Proc. ICEE, (2006).

[21] J. G. ProAKIS, Digital Communications. New York: McGraw-Hill, (2001).

[22] R. Johnson, P. SCHNiter, T. EndRES, J. BEHM, D. BROWN R. CASAS, Blind equalization using the Constant Modulus criterion: A review. Proc. IEEE, 86 (1998), pp. 1927-1950.

[23] D. Stranneby, Digital Signal Processing: DSP and applications. MATLAB Neural Network Toolbox User's guide, (2002).

[24] P. McCullagh, Tensor Methods in Statistics. Chapman \& Hall, (1987). 
[25] C. CoRTeS, V. VAPNIC, Support vector network. Machine Learning, 20 (1995), pp. 1-25.

[26] C. Burges, A tutorial on support vector machines for pattern recognition. Data Mining and Knowledge Discovery, 2 (1998), pp. 121-167.

[27] B. E. BosER, V. VAPNIK, A training algorithm for optimal margin classifiers. 5th ACM, (1996).

[28] P. HOEHER, A statistical discreet-time model for the WSSUS multipath channel, IEEE Trans. Vehicular Tech., 41 (1992), pp. 461-468.

[29] S. Chen, C. F. N. CowAn, P. M. GRANT, Orthogonal least squares learning algorithm for radial basis networks. IEEE Trans. Neural Networks, 2 (1991), pp. 302-309.

Received: May, 2006

Revised: April, 2007

Accepted: April, 2007

Contact addresses:

Ataollah Ebrahimzadeh

Faculty of ECE

Noushirvani Institute of Technology

Research Group on Signal Processing and Pattern Recognition

Shariati Blvd, Babol, Iran

e-mail: ataebrahim@yahoo.com, e_zadeh@nit.ac.ir

GholamReza Ardeshir Faculty of ECE

Noushirvani Institute of Technology Research Group on Signal Processing and Pattern Recognition Shariati Blvd, Babol, Iran e-mail: g.ardeshir@nit.ac.ir
ATAOLLAH EBRAHIMZADEH works at the Faculty of Electrical and Computer Engineering at Noushirvani Institute of Technology, Babol. His areas of interest include wireless communications, statistical pattern recognition and signal processing.

GHOLAMREZA ARDESHIR works at the Faculty of Electrical and Computer Engineering at Noushirvani Institute of Technology, Babol. His areas of interest include statistical pattern recognition and artificial intelligence. 